\title{
Arrival Trajectory Optimization on Noise Impact using Interval Analysis
}

\author{
H. $\mathrm{Yu}^{*}$ and J. A. Mulder ${ }^{\dagger}$ \\ Delft Univeristy of Technology, Kluyverweg 1, 2629 HS Delft, The Netherlands.
}

\begin{abstract}
The CleanEra project is initiated by the Faculty of Aerospace Engineering at Delft University of Technology. Significant reduction of noise impact on perimeters of airports via arrival trajectory optimization is considered as one of the major targets of the project. In this paper, an aircraft model and its acoustic performance are introduced to assist the development of the optimization tool since the CleaenEra project is still ongoing. Interval analysis is explained in details because interval algebra is employed to deal with real intervals rather than real numbers in the so-called interval-related optimization algorithms. The largest advantage of such algorithms is that they are capable of finding the global optimum out of the multiple local minima to a given optimization problem. Two numerical examples are presented to show the usefulness of such algorithms while solving both static and dynamic optimization problems. In the end, a third example is given to demonstrate the applicability of the proposed optimization tool to trajectory optimization for arriving passenger aircraft.
\end{abstract}

\section{Nomenclature} flight altitude true airspeed heading angle angle of attack bank angle closed real interval time derivative

horizontal distance from the aircraft to the runway threshold lateral distance from the aircraft to the centerline of the runway flight-path angle which is negative during arrival thrust of the engines along the $x$-axis of the body-fixed coordinate system aerodynamic lift force aerodynamic drag force mass of the aricraft assumed to be constant gravitational acceleration scalar cost function of the optimization problem vector of state variables of the optimization problem vector of control variables of the optimization problem vector of constraints of the optimization problem infimum of the closed real interval infimum of the closed real interval parameters in the optimization problem

*Ph.D student, Control and Simulation Division, Faculty of Aerospace Engineering, AIAA Young Professional Member. ${ }^{\dagger}$ Professor, Control and Simulation Division, Faculty of Aerospace Engineering, AIAA Member. 


\section{Introduction}

As one of the environmental issues that have top priorities, noise nuisance of passenger aircraft has become one of the most essential concerns of air transport and aircraft operations. Traditionally, the reduction of noise at source can be achieved by phasing out the aircraft that fall short of the adopted certification standards. Basing upon the available technologies, the International Civil Aviation Organization (ICAO) Council updates the certification limits without compromising the efficiency of air transport, the safety of aircraft operations, and a number of other environmental issues in terms of noise nuisance and fuel consumption. Included in Chapter 2 of Annex 16, ${ }^{1}$ the first standards were initiated for subsonic jet-powered aircraft, for which the application for a type certificate was submitted before 6 October 1977. Subsequently, due to the developed technologies of airframe and engine design, more stringent standards corresponding to Chapter 3 of Annex 16 were set and targeted for subsonic jet-powered aircraft, for which a type certification was requested on or after 6 October 1977 and before 1 January 2006. On 1 January 2006, the latest and strictest standards, listed in Chapter 4 of Annex 16, became applicable to newly certificated subsonic jetpowered aircraft. Next to these standards, the ICAO Council introduced the concept of Balanced Approach to address the problem issue in the most cost-effective manner since a research conducted by the ICAO Council's Committee on Aviation Environmental Protection (CAEP) concluded that the additional phaseout would only have a very limited environmental benefit and the cost would be extremely high. Involved in the concept are four elements as follows:

1. reduction of noise at source;

2. land-use planning and management;

3. noise abatement operational procedures; and

4. operating restrictions on aircraft.

Accordingly, the ICAO developed specific policies and guidance for the proper implementation of the Balanced Approach and all ICAO members are urged to fully implement this advanced concept. The principle behind the Balanced Approach is that solutions need to be adjusted based on the specific characteristics of the airport under consideration. In response to this principle, we carry out a research on noise abatement operational procedures which are designed not only for a particular airport but also a particular aircraft. That is, both the conditions around the airport and the performance capabilities of the aircraft are taken into account during the design of such operational procedures.

\section{A. The CleanEra Project}

Initiated by the Faculty of Aerospace Engineering at Delft University of Technology, the CleanEra ${ }^{2}$ project aims at developing new technologies optimized for revolutionary conceptual aircraft designs for environment and passenger friendliness and at investigating the feasibility of these technologies and their integration. As one of the major targets of the project, significant reduction of noise nuisance is the focus of this paper. By optimizing arrival trajectories with the expectation of minimizing the noise impact on the perimeter of the operating airport, a set of noise abatement operational procedures can be achieved for the CleanEra aircraft.

Meantime, the CleanEra project also covers a number of other research topics, such as aerodynamics, materials, structure, propulsion, acoustics, and so on. The difference between the topics in the list and the one presented in this paper is that the former take into account a single or multiple components of the aircraft design while the latter focuses on arrival trajectory optimization after the aircraft has already been assembled. In other words, the technology being developed by the authors is applicable for both existing and upcoming aircraft as long as their performance capabilities are known from flight tests or predicted by flight simulations.

\section{B. State of the Art}

With a traditional step-down approach trajectory, an arriving passenger aircraft descends from the Initial Approach Fix (IAF) to reach a certain altitude and then performs a horizontal flight segment to intercept with the final three-degree glide-slope at the Final Approach Fix (FAF). The disadvantage of this approach is that the aircraft executing the horizontal flight segment at low airspeeds extends its flaps to generate a sufficient aerodynamic lift force and thus requires high thrust settings to overcome the large aerodynamic drag force. Besides the extra fuel consumption, the aircraft causes more noise nuisance due to the fact that the aircraft operates with such high thrust settings at such low altitudes. In order to reduce the noise 
nuisance as well as the fuel consumption of the aircraft within the Terminal Maneuvering Area (TMA), the horizontal flight segments at low altitudes should be minimized or even completely avoided and the engines of the aircraft should operate at relatively low thrust settings as long as possible.

It has been shown that noise abatement arrival procedures can be achieved with the assist of tools for communication and navigation, such as the Continuous Descent Approach ${ }^{3}$ (CDA) and the Three-Degree Decelerating Approach ${ }^{4}$ (TDDA). Developed to improve the conventional step-down approach trajectories, both of these two procedures aim at eliminating the horizontal flight segments in order to avoid high thrust settings at low altitudes. During the development of these arrival procedures, however, advanced optimization algorithms are not employed to optimize the entire flight profile in order to find the local or global minimum value of the noise nuisance caused by an arrivaing passenger aircraft. Choosing a trajectory which minimizes or maximizes a certain cost function is referred to as trajectory optimization. Visser and Wijnen ${ }^{5}$ presented an optimization tool for analysis and design of noise abatement arrival procedures, in which a dynamic trajectory optimization algorithm is combined with an aircraft model, a noise model, and a geographic information system. The optimization is carried out using an optimization package that implements a simple variant of the collocation method. Although the results show that the overall reduction of noise nuisance has already been quite encouraging, more improvements are still expected if advanced global optimization algorithms are employed to solve such a highly nonlinear dynamic optimization problem.

As for an optimization problem with multiple local optima, conventional optimization algorithms can not guarantee finding the global optimum when the search process is terminated by certain stopping criteria. The reason can be roughly explained as follows. These conventional algorithms select a set of sample points in certain ways and the local or global optima are considered to be among these sampled points. Note that the number of the sampled points is always limited and thus the opportunity of missing the local or global optima during the sampling can not be ignored. Differently, optimization algorithms developed based on interval analysis do not sample at individual points but evaluate a continuum of points which can be called a set. Interval analysis has been attracting wide attention since it was introduced by Moore ${ }^{6}$ in 1966, although it was only transformed into a tool to bound the effect of errors at the very beginning of its period. Between 1979 and 1980, optimization algorithms were built by Hansen ${ }^{7,8}$ to provide guaranteed bounds on the global optima for both one-dimensional and multi-dimensional cases. The basic principles behind such algorithms are following:

1. interval algebra is used to transform traditional discrete value operations into their interval counterparts;

2. the initial design domain(s) is split into a finite number of sub-domains in such a way that all the points in the design space are evaluated during the optimization process; and

3. the domain(s) containing the global optimum is found and reduced in size until the required accuracy is obtained.

For the sake of simplicity, optimization algorithms developed based on interval analsyis are called intervalrelated algorithms in this paper. Due to the very principles mentioned above, such algorithms have become quite preferable and been successfully applied in an increasing number of fields. There are a few examples in the field of aerospace engineering, including both aeronautics and astronautics: nonlinear aircraft trimming, ${ }^{9}$ pilot model parameters identification, ${ }^{10}$ Terminal Area Energy Management (TAEM) trajectory optimization for Reusable Launch Vehicles (RLVs), ${ }^{11}$ spacecrafts formation rotations,${ }^{12}$ and so on.

From the foregoing analyses, we may conclude that the potential of reducing the noise nuisance of an arriving passenger aircraft using interval-related optimization algorithms is obvious and attractive. In order to realize this potential, an optimization tool combined with a particular aircraft model and the acoustic performance of the selected aircraft is proposed in this study. Meanwhile, the population distribution model around an airport is also built during the stage of modeling since awakenings rather than noise footprints is selected as the cost function of the optimization problem.

\section{Structure of the Paper}

This paper is structured in the following way. In Section II, the optimization problem is stated, including the aircraft model, the two-dimensional point-mass equations of motion, the initial and final conditions of the trajectory, and the cost function selected to be minimized in the process of optimization. In Section III, interval analysis is brought in as a preparation of the introduction of the interval-related algorithms. Moreover, two simple examples are given to demonstrate the search process in details. Section IV presents 
three numerical examples in total. The first two are taken from previous publications while the third one is about a part of an arrival trajectory in practice. Results are presented to prove that the proposed intervalrelated algorithms guarantee finding the global optimum for both static and dynamic optimization problems and that the proposed optimization tool is applicable for optimizing trajectories for arriving passenger aircraft. Finally, conclusions are drawn in Section V.

\section{Problem Statement}

In this paper, trajectories of arriving passenger aircraft are optimized with the expectation of minimizing the noise impact on the perimeter of the operating airport. Note that we only itemize the issues that are fully necessary in the optimization process.

\section{A. Flight Envelope}

Within the phase of arrival, the pilots in the cockpit are required to extract flaps and landing-gears based on the indicated airspeed of the aircraft. Therefore, an aircraft model is required to provide the indicated airspeed schedule for extending the flaps and landing-gears as a reference; the point-mass equations of motion of such an aircraft are inevitable in order to generate trajectories that are physically achievable; initial and final points of the arrival determine the start and the end of the flight simulation, respectively.

\section{Aircraft Model}

The CleanEra project is still ongoing and thus the performance capabilities of the CleanEra aircraft are unknown yet. In order to proceed further, an existing aircraft model at a similar scale has to be selected to assist the development of the optimization tool. In this study, we choose a Boeing 747-400 aircraft model ${ }^{13}$ with four advanced high-bypass engines installed.

\section{Point-Mass Equations of Motion}

In practice, trajectories of arriving passenger aircraft are designed within a three-dimensional TMA, in which the aircraft are delivered safely and efficiently from the IAF to the FAF. In order to simplify the problem at the beginning stage of this research, we are only concerned with a two-dimensional TMA instead of a threedimensional one. Furthermore, it is assumed that all the forces acting on the aircraft go through its center of gravity, as can be seen in Fig. 1. From that point on, the weight points to the ground perpendicularly; the aerodynamic lift force is perpendicular to the direction of the speed and pointing up; the aerodynamic drag force points along the opposite direction of the speed; and the thrust is along the $x$-axis of the body-fixed coordinate system. Therefore, the state vector of the system reduces from $\left[x, y, h, V_{T}, \gamma, \chi\right]^{T}$ to $\left[x, h, V_{T}, \gamma\right]^{T}$ while the control vector from $[\alpha, \mu]^{T}$ to $[\alpha]^{T}$. The point-mass equations of motion can be derived and written in the form of:

$$
\left\{\begin{array}{rl}
\frac{d x}{d t} & =V_{T} \cos \gamma \\
\frac{d h}{d t} & =V_{T} \sin \gamma \\
\frac{d V_{T}}{d t} & =\frac{T\left(V_{T}, h\right) \cos \alpha-D\left(V_{T}, h, \alpha\right)-m g \sin \gamma}{m} \\
\frac{d \gamma}{d t} & =\frac{T\left(V_{T}, h\right) \sin \alpha+L\left(V_{T}, h, \alpha\right)-m g \cos \gamma}{m V_{T}}
\end{array} .\right.
$$

In Eq. 1, the aerodynamic lift and drag forces are functions of the true airspeed, the flight altitude and the angle of attack of the aircraft. In this research, the engine thrust is set to be flight-idle or nearly flight-idle during the entire flight in order to minimize the fuel consumption as well as its noise emission. Although it is easy to observe that the control input of the optimal control problem is the angle of attack, the actual control of the aircraft is achieved by moving some or all of its control surfaces. The connection between the required angles of attack and the movements of the control surfaces is not the focus of this research. As long as the angles of attack vary in the ranges that are allowed by the performance capabilities of the aircraft, this connection can be realized physically. 


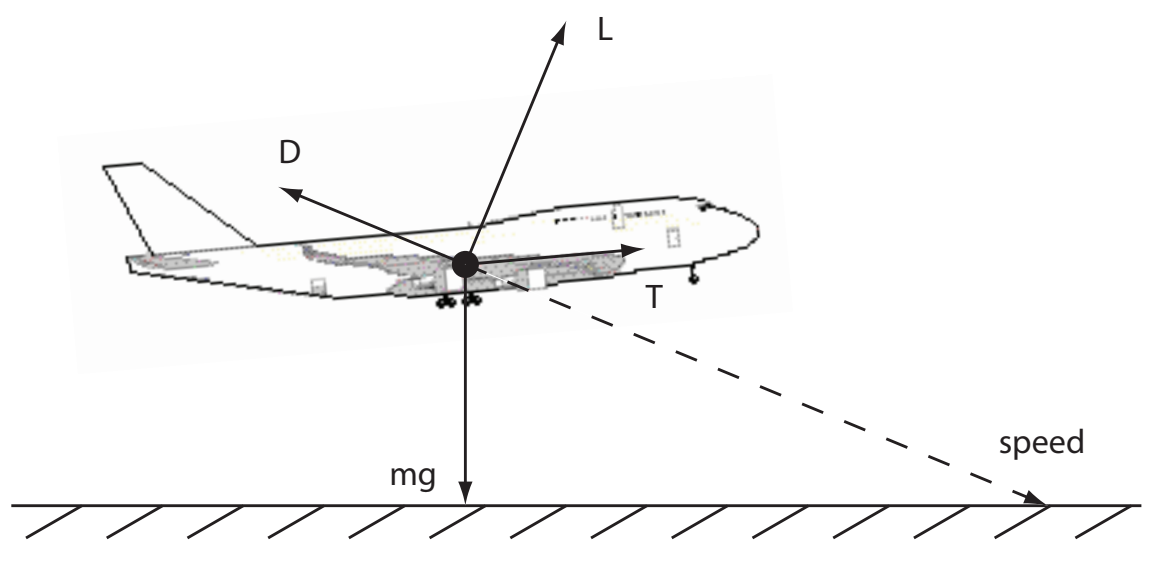

Figure 1. All the forces acting on the aircraft and the direction of the speed.

\section{Boundary Conditions}

At the beginning section of the phase of arrival, the airspeed of the aircraft is so high that the pilots do not extend the landing-gears or any flaps. Plus, the distance from the aircraft to the ground is so large that the aircraft is inaudible and thus the aviation noise at this section is not considered as acoustic pollution. Therefore, the arrival trajectory reviewed in this research starts from the IAF to the FAF, which represents the last flight segment before the three-degree glide-slope. Similar with the traditional step-down approach, the aircraft intercepts the Instrument Landing System (ILS) at the FAF and then navigates along the three-degree glide-slope to the threshold of the runway. Accordingly, the initial and final conditions of the optimization problem are the states of the aircraft at IAF and FAF, respectively. Details can be seen in Fig. 2. The final three-degree glide-slope is extended backwards till intercepting with FL100. At different airports, there are different restrictions on the position of IAF so that there are at lease two possible variations: (1) the horizontal distance varies while the altitude remains constant; or (2) the horizontal distance stays constant while the altitude changes. In this research, the point on the extension line is selected as the IAF in order to compare the results obtained by the proposed optimization tool with that from the TDDA.

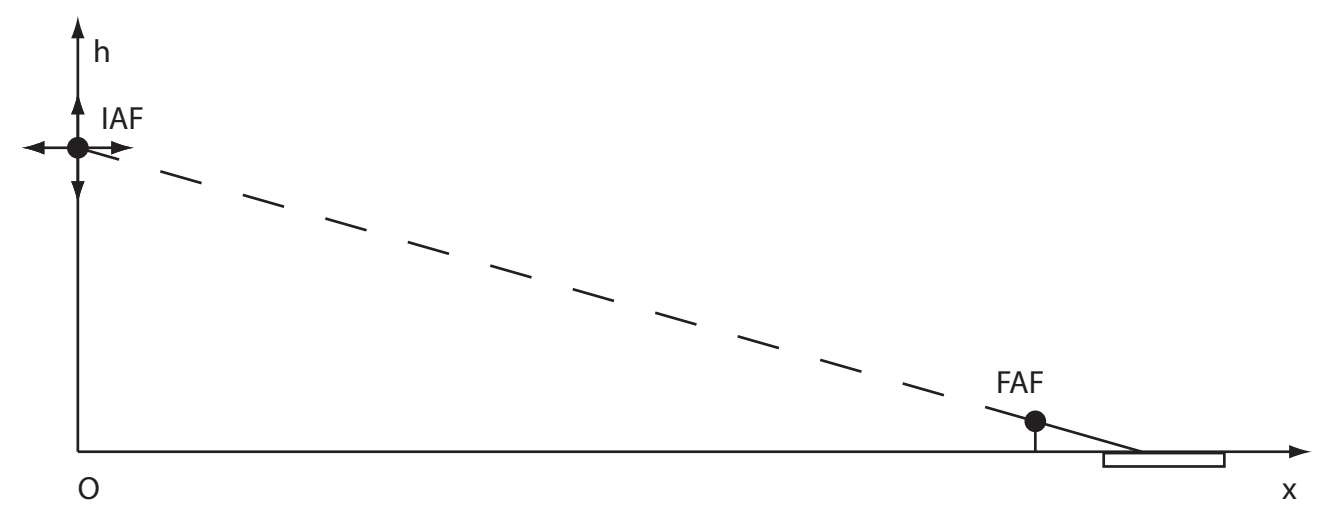

Figure 2. Initial and final points of a two-dimensional arrival trajectory.

\section{B. Cost Function}

There exist quite a lot of metrics to assess the responses of people to aviation noise impacts, such as speech interference, sleep interference, hearing loss, and so on. Most of these metrics are influenced by both emotional and physical variables and thus difficult to be simply represented by mathematical expressions. 


\section{Noise Model}

Noise emission generated by the selected aircraft is calculated based on the Integrated Noise Model ${ }^{14}$ (INM) which is developed by the Federal Aviation Administration (FAA). It is important to note that the noise levels at regularly distributed observing points are obtained based on the combination of the Noise-PowerDistance (NPD) datasets and particular environment-related adjustments. Given the speed profile of the aircraft, the thrust settings of the engines, the distances from the aircraft to the observing points and the environmental conditions as inputs, noise levels at those observing points can be returned by the INM as outputs. With these outputs, noise levels at certain measuring points or noise footprints on the ground can be obtained to demonstrate the noise nuisance of a flyover passenger aircraft.

\section{Awakenings}

Traditionally, the area of a footprint at a certain level is selected as the cost function of the optimization problem. In this case, there exists a risk that some uninhabited places are enclosed by the footprint and the minimization of its area has no practical benefit to the people who are living in the operating TMA. In order to overcome this defect, a concept called awakenings is introduced to combine the noise levels and the people encountering the noise emissions in the TMA. A dataset of the population distribution in the TMA is necessarily required in order to implement the concept of awakenings. For the purpose of simulation, such a dataset can be built by dividing the entire area into either regular or irregular sub-areas based on the actual situation. Each of these sub-areas can be represented by one observing point inside with its $x$ - and $y$ - coordinates in the Earth-fixed coordinate system. Importantly, a third dimension has to be added to hold the number of people enclosed by that certain sub-area. Since the sub-area is always small, it is reasonable to assume that the noise levels at all points inside are identical with that at the selected observing point.

The overall number of awakenings can be obtained as long as the indoor Sound Exposure Levels (SELs) at all observing points are returned from the noise model. The relationship between the Percent Awakening (\%Awakening) and the indoor $\operatorname{SEL}\left(S E L_{\text {indoor }}\right)$ can be seen in Fig. 3, in which all the circles are statistical data points from field studies and the dashed line is an interim sleep disturbance dose-response relationship recommended by the Federal Interagency Committee On Noise (FICON) in 1992. In 1997, the Federal Interagency Committee on Aviation Noise (FICAN) updated the relationship ${ }^{15}$ whose mathematical expression is as follows:

$$
\% \text { Awakenings }=0.0087\left(S E L_{\text {indoor }}-30\right)^{1.79}
$$

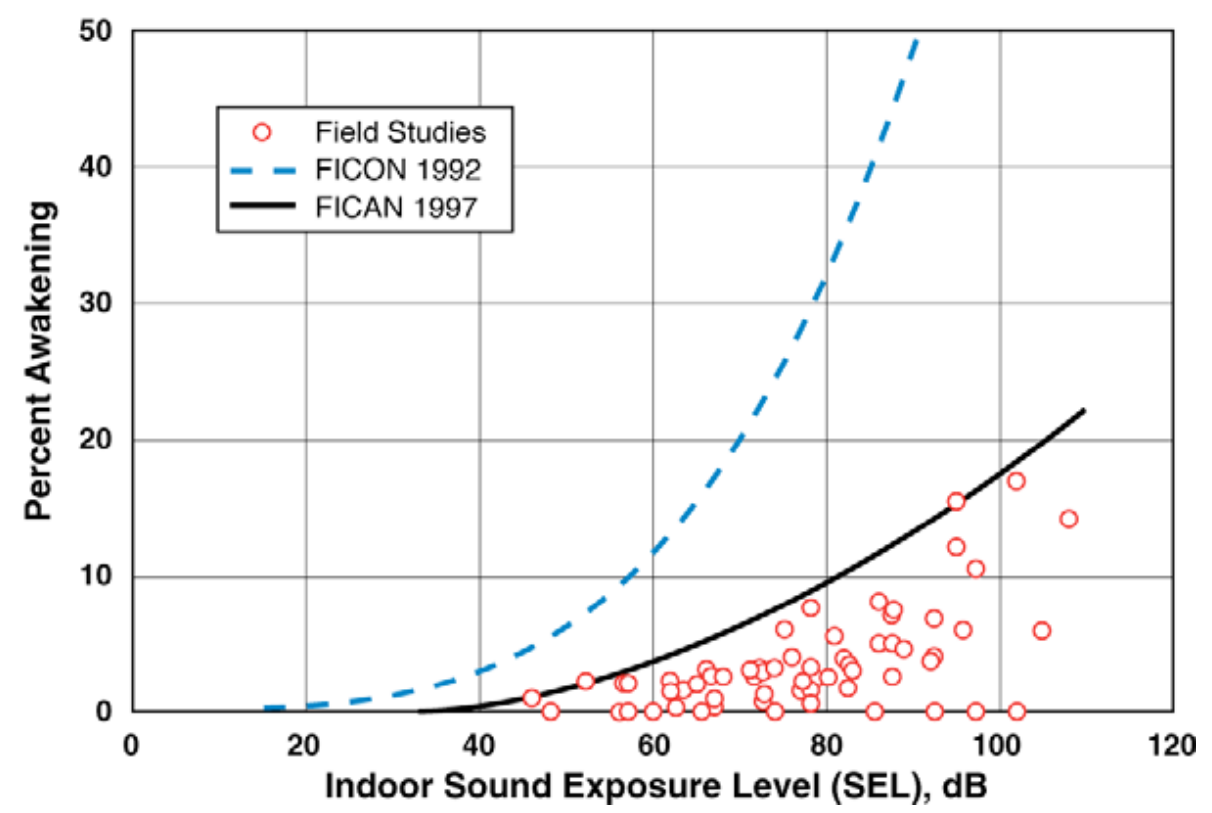

Figure 3. Recommended sleep disturbance dose-response relationship by the FICAN in 1997. 


\section{Optimization Problem}

After all the preparations, a highly nonlinear dynamic optimization problem can be generated in the form of:

$$
\min \{J(\mathbf{x}, \mathbf{u})\}
$$

subject to

$$
\mathbf{x}, \mathbf{u} \in \mathbf{G},
$$

where the optimal solution of the control vector $\mathbf{u}$ has to be found to generate the optimal trajectory of the state vector $\mathbf{x}$ that minimizes the cost function $J(\mathbf{x}, \mathbf{u})$ while satisfying all the constraints represented by $\mathbf{G}$.

The complete optimization tool should consist of portable modules so that different aircraft models, noise models, population distribution models, and sleep disturbance relationships can be integrated and segregated easily as the users' wish.

\section{Interval Analysis}

In this section, we summarize the definition of intervals, interval operations, interval functions, the concept of consistency and finally the interval-related algorithms.

\section{A. Interval Arithmetic}

A closed real interval is a set of consecutive real numbers that are enclosed by two endpoints. The endpoint on the left is referred to as the infimum of the interval and the one on the right is called the supremum of the interval. Denote the set that includes all the real numbers by $\mathbb{R}$. Then a closed real interval $P$ can be written as:

$$
P=[\underline{P}, \bar{P}]=\{p \in \mathbb{R}: \underline{P} \leq p \leq \bar{P}\},
$$

in which $\underline{P}$ and $\bar{P}$ are the infimum and the supremum of $P$, respectively. Note that the terms 'real interval' and 'interval' are used interchangeably in this paper since no complex intervals are involved in the optimization process.

\section{Interval Operations}

The basic operations for real arithmetic $(+,-, \times, \div)$ can be extended to interval arithmetic in terms of operations on the infima and suprema of the given intervals. If we denote any one of the basic operations for interval arithmetic by ' $\odot$ ', then the calculation can be defined as:

$$
X \odot Y=\{x \odot y: x \in X, y \in Y\},
$$

in which $X$ and $Y$ are both closed intervals. Details of the four basic operations for interval arithmetic can be expressed as follows:

$$
\left\{\begin{array}{rl}
X+Y & =[\underline{X}+\bar{Y}, \bar{X}+\underline{Y}] \\
X-Y & =[\underline{X}-\bar{Y}, \bar{X}-\underline{Y}] \\
X \times Y & =[\min (\underline{X Y}, \underline{X} \bar{Y}, \bar{X} \underline{Y}, \overline{X Y}), \max (\underline{X Y}, \underline{X} \bar{Y}, \bar{X} \underline{Y}, \overline{X Y})] \\
X \div Y & =X \times\left[\frac{1}{\bar{Y}}, \frac{1}{\underline{Y}}\right] \text { if } 0 \notin Y
\end{array} .\right.
$$

If zero is included in $Y$, then the rule for the operation of division has to be extended by involving infinite or semi-infinite intervals. Provided that $X$ is equal to $[2,4]$, an infinite interval is retuned when $Y$ equals $[-1,1]$ :

$$
X \div Y=[2,4] \div[-1,1]=[-\infty, \infty]
$$

and a semi-infinite interval is obtained when $Y$ is equal to $[0,1]$ :

$$
X \div Y=[2,4] \div[0,1]=[2, \infty] .
$$

In the optimization process, the appearance of such infinite or semi-infinite intervals is not preferable since the final goal is to reduce the sizes of the intervals as fast as possible. 


\section{Interval Functions}

An interval-valued function of one or more than one interval variable is referred to as an interval function. To demonstrate an important nature of interval functions, we consider $F$ as a function of a finite number of interval variables $X_{1}, \ldots, X_{n}$. If $F$ combines all the interval variables with the basic operations for interval arithmetic, then $X_{i} \subseteq Y_{i}(i=1, \ldots, n)$ implies:

$$
F\left(X_{1}, \ldots, X_{n}\right) \subseteq F\left(Y_{1}, \ldots, Y_{n}\right),
$$

which is called inclusion theorem and considered as the core theorem in the application of interval analysis.

Evaluations of an interval function provide results that guarantee containing all possible values of the solution. However, the results may also contain values that do not belong to the exact solution set. This phenomenon is called overestimation, which is always a result of the dependency problem and the wrapping effect as explained below:

- The dependency problem exists because the basic operations for interval arithmetic can not identify multiple appearances of a single interval variable. Take the operation of subtraction as an example. Given $X=[2,4]$, the result of $X-X$ is $[-2,2]$ instead of $[0,0]$. That is, the subtraction is treated as $X-Y$ with $X=[2,4]$ and $Y=[2,4]$. As a result, the result is significantly overestimated.

- Generally, a set of intervals can be grouped into an interval vector. The image of an interval vector under some certain mappings may not be an interval vector any more. In this case, the results would be overestimated if the mapped image had to be wrapped with an interval vector. This phenomenon is called the wrapping effect.

In interval calculations only involving the four basic operations mentioned above, the dependency problem makes the resulting intervals unexpectedly wider than the exact ones. Therefore, if possible, we should rewrite the expressions in such a way that the times of the appearance of a single interval variable are maximally reduced. Differently, the wrapping effect usually occurs in interval integrations so that the integrals blow up very fast. One way of avoiding such a fatal effect is to avoid integrations at all in interval calculations.

\section{Concept of Consistency}

As stated previously, the dependency problem and the wrapping effect overestimate the intervals obtained from interval calculations. In order to overcome this obstacle, the concept of consistency ${ }^{16,17}$ is introduced and implemented. There are quite a number of procedures derived from this concept which can successfully sharpen large intervals. In this section, we just present the hull consistency as an example. It is assumed that a function has the form of:

$$
f(x)=g(x)-h(x)=0
$$

with its solution $x^{*}$ contained by the initial interval $X$. If $g(x)$ is invertible, then we can solve the equation to obtain $x=g^{-1}[h(x)]$. According to the inclusion theorem, we have:

$$
x^{*}=g^{-1}\left[h\left(x^{*}\right)\right] \in g^{-1}[h(X)] .
$$

Therefore, the initial interval $X$ may be shrunk or eliminated by this repeatable process since the solution of the function is contained in the newly formed interval $X^{\prime}=X \cap g^{-1}[h(X)]$. Following are the three possibilities after applying the hull consistency:

- If $X^{\prime}$ is equal to $X$, then the result is not improved at all.

- If $X^{\prime}$ is smaller than $X$ but not empty, then the initial interval is sharpened.

- If $X^{\prime}$ is empty, then the initial interval can be safely discarded. The function has no solution at all.

\section{B. Interval-Related Algorithms}

From the previous analysis, we easily realize that both the interior and final results are intervals when interval-related algorithms are employed, which is one of the differences between such advanced algorithms and other conventional ones. 


\section{Optimization Algorithms}

In a branch-and-bound algorithm, after applying a splitting procedure to split the initial design domain(s) into smaller sub-domains, a bounding procedure follows to compute the lower and upper bounds of the cost function for all the resultant sub-domaines and some of these sub-domains can be shrunk or eliminated through a procedure called pruning. Interval-related optimization algorithms are developed based on the same principles. The flowchart of such algorithms is shown in Fig. 4 and the algorithm-steps are listed roughly as follows:

1. Begin with the initial design doamin(s);

2. Split the remaining domain(s) into smaller sub-domains in a defined manner;

3. Shrink the sub-domains that may contain the global optimum and eliminate those that surely do not contain the global optimum; and then

4. Iterate step 2 and step 3 until the stopping criteria are satisfied. If the optimization is solved successfully, a sufficiently small set of points (a single domain or multiple sub-domains) remains. Then the global optimum is guaranteed to be in the remaining doamin(s).

The optimization algorithm normally begins its search in one domain. In practice, they may also start with more than one domain wherein the global minimum is possibly located. There is no disadvantage if the search space formed by these multiple domains is not simply connected.

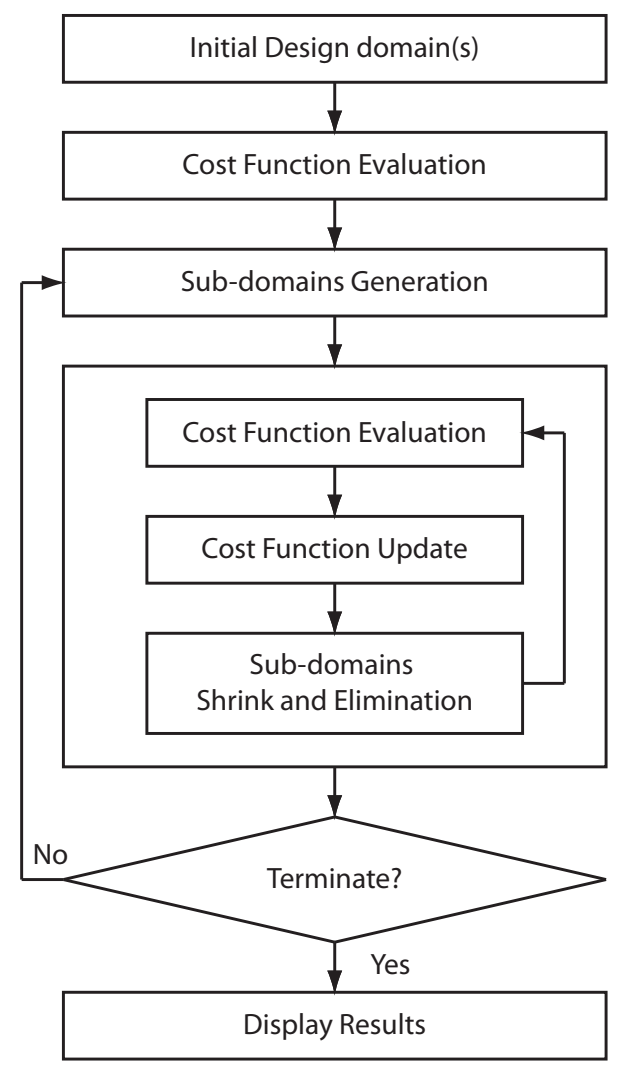

Figure 4. Flowchart of the proposed interval-related algorithms.

\section{Demonstration}

Among all the steps, how to find and discard the sub-domain(s) that surely does not contain the global optimum is the focus point. Following are two examples that demonstrate the elimination of the so-called unfeasible sub-domain(s). 
A one-dimensional optimization problem is represented by minimizing the value of a function with one variable. The function has the form of:

$$
f(x)=x^{4}-10 x^{2}+x+1,
$$

where the initial interval for the only variable $x$ is $[-3,1]$. As we can see in Fig. 5, the initial interval is firstly divided into eight sub-intervals and their widths are all the same since they are averagely subdivided. Denote the sub-intervals along the $x$-axis from left to right by $x_{1}, \ldots, x_{8}$ and the corresponding results of the function obtained from interval calculations by $f_{1}, \ldots, f_{8}$. We take $x_{2}$ and $f_{2}$ as an example. In $x_{2}$, it is easy to notice that the lower bound of $f_{2}$ is smaller than the minimum value that the curve is able to reach and that the upper bound of $f_{2}$ is greater than the maximum value that the curve is able to reach. This phenomenon is due to the dependency problem and applies to the other sub-intervals as well. However, the upper bound of $f_{2}$ can be set as the temporarily minimum value of the function, which will obviously be updated in the following iterations. It is greatly useful at this moment because sub-intervals $x_{5}, \ldots, x_{8}$ can be safely eliminated due to the fact that the minimum value enclosed by $f_{5}, \ldots, f_{8}$ is greater than the temporary minimum. The next iterations will spilt the remaining sub-intervals $x_{1}, \ldots, x_{4}$ into smaller ones and the same procedures will be carried out till the required accuracy is obtained.

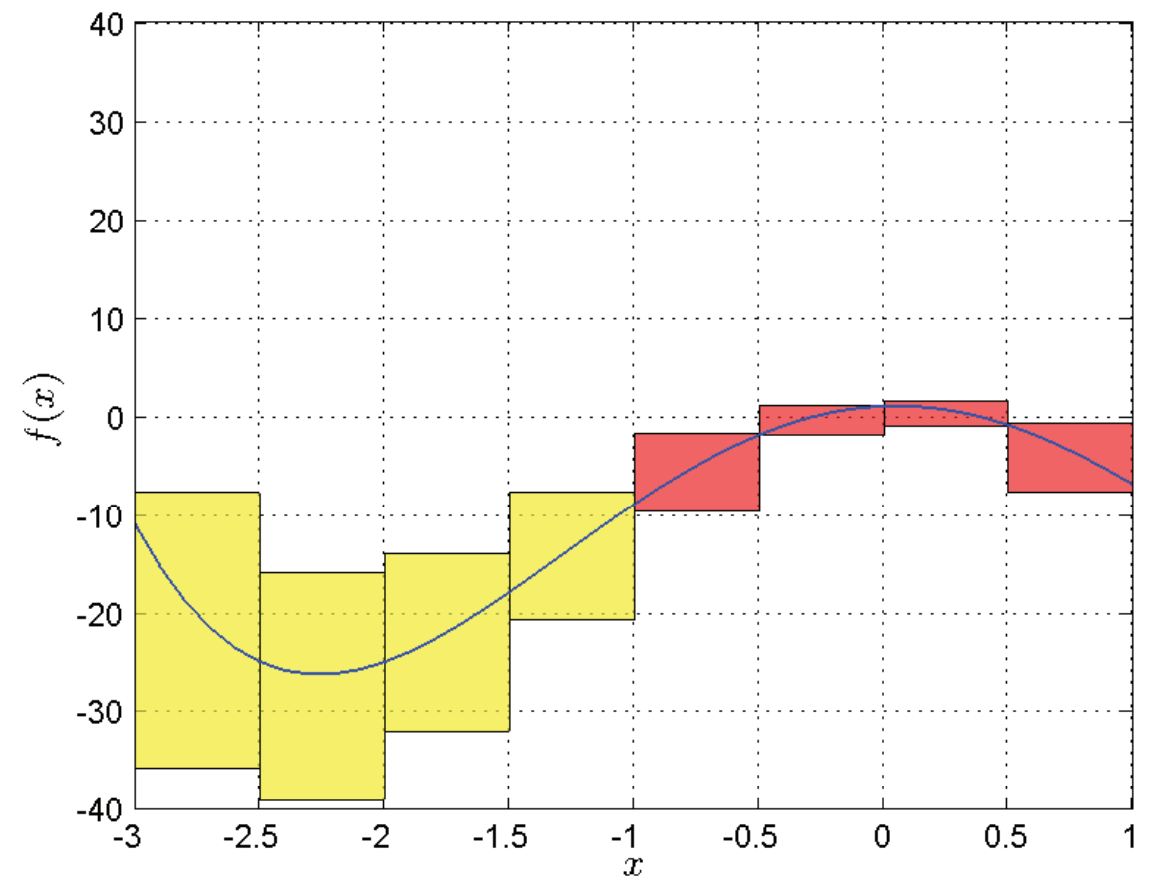

Figure 5. Sub-intervals and sub-boxes generation and elimination.

A two-dimensional optimization problem is represented by minimizing the value of a function with two variables. The function has the form of:

$$
f\left(x_{1}, x_{2}\right)=x_{1}^{3}-10 x_{1}+5 x_{1} x_{2}-2 x_{2}-x_{2}^{3},
$$

where $x_{1}$ and $x_{2}$ have the same initial interval $[-2,2]$. Denote a domain that is enclosed by two intervals by box. For the sake of simplicity during explanation, only three boxes are selected to demonstrate the processes of eliminating unfeasible sub-boxes and they are named by $b_{1}, b_{2}$, and $b_{3}$ from left to right. The results of the function obtained through interval calculations are name by $f_{1}, f_{2}$ and $f_{3}$, correspondingly. Details are shown in Fig. 6. Merely considering those three boxes, the upper bound of $f_{3}$ can be set as the temporarily minimum value of the function and thus the box $b_{1}$ can be safely discarded since the lower bound of $f_{1}$ is slightly greater than the temporary minimum. The remaining boxes will be split and eliminated again and again in following iterations till the stopping criteria are satisfied. 


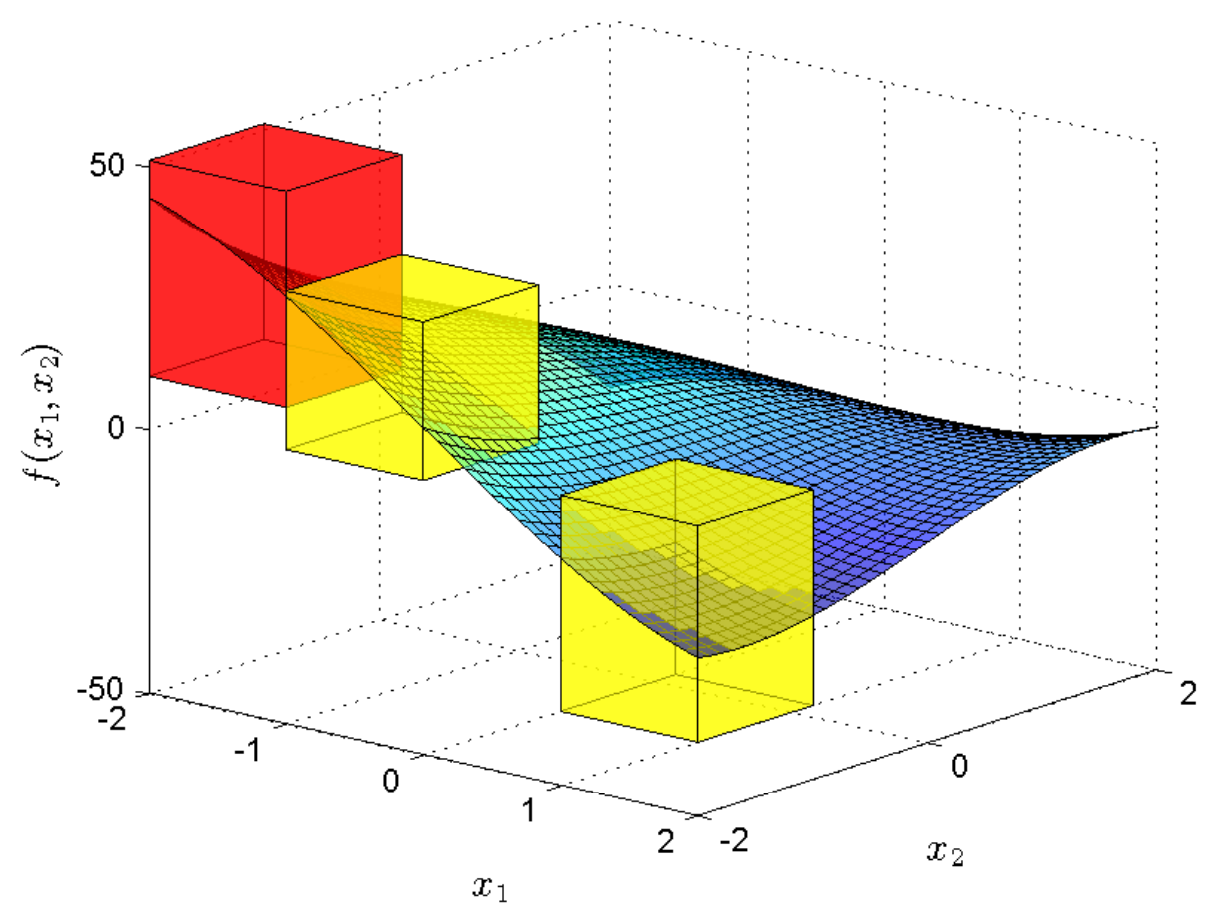

Figure 6. Sub-intervals and sub-boxes generation and elimination.

Although it is difficult to demonstrate with figures, such interval-related algorithms are still applicable for practical optimization problems with more than two dimensions.

\section{Numerical Results}

In this section, three numerical examples are given to demonstrate the application of the interval-related algorithms. An interval arithmetic toolbox, INTLAB, ${ }^{18,19}$ is used to build the algorithms.

\section{A. Example 1}

In order to show that the interval-related algorithms have the capability of finding the global minimum, a static optimization problem with multiple local minima is selected as the first example.

The goal of this optimization problem is to minimize the value of a function with the form of:

$$
f(x)=1+\left(x^{2}\right)^{0.25} \times\left\{1+\sin ^{2}\left[50\left(x^{2}\right)^{0.1}\right]\right\},
$$

in which the single variable $x$ varies within a given design space $[-100,+100]$. Let $f^{*}$ denote the global minimum and $x^{*}$ denote the point at which $f^{*}$ occurs. From the curve of the function shown in Fig. 7 , we notice that this optimization problem has a series of local optima and the smallest one of them is the global minimum. From analytical calculations, we have:

$$
\left\{\begin{array}{l}
x^{*}=0 \\
f^{*}=1
\end{array} .\right.
$$

As mentioned in the previous sections, the interior and final results are both intervals instead of real numbers so that the stopping criteria have to be given to constrain the widths of the resulting intervals. In this example, the optimization process terminates when the widths of the intervals for the variable and the function value are both smaller than $1.00 \times 10^{-6}$. As can be seen in Table 1 , the width of the interval for the variable is about $0.52 \times 10^{-6}$ while the width of the interval for the function value is around $5.267 \times 10^{-13}$. 


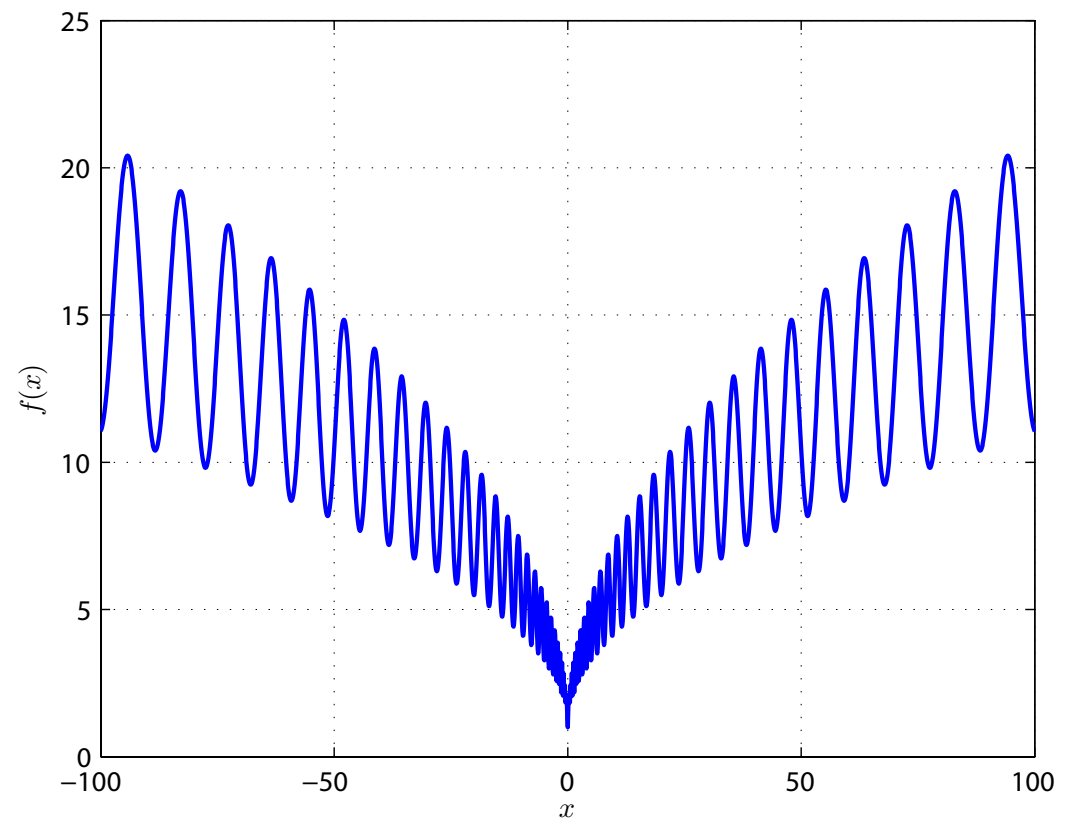

Figure 7. Example 1: a static optimization problem with multiple local minima.

Table 1. Interior and final results of Example 1.

\begin{tabular}{ccc} 
Iteration & Interval for $x$ & Interal for $f$ \\
\hline 1 & {$[-27.2727273,27.2727273]$} & {$[1.00000000,4.54081192]$} \\
2 & {$[-2.47933885,2.47933885]$} & {$[1.00000000,2.55756065]$} \\
3 & {$[-0.37565741,0.37565741]$} & {$[1.00000000,1.54749436]$} \\
4 & {$[-0.02049041,0.02049041]$} & {$[1.00000000,1.09550756]$} \\
$\vdots$ & $\vdots$ & $\vdots$ \\
14 & {$\left[-2.562 \times 10^{-13}, 2.705 \times 10^{-13}\right]$} & {$[1.00000000,1.00000052]$}
\end{tabular}

\section{B. Example 2}

In order to use interval-related algorithms as direct methods to solve a dynamic optimization problem, it is necessary to first convert that into a static optimization problem. In the following example, the conversion is carried out by parameterizing the control function and rewriting all the other functions with the introduced parameters.

The cost function of the original dynamic optimization problem is expressed as follows:

$$
J=\int_{1}^{0}\left[0.5 u^{2}(t)+x(t)\right] d t
$$

and the dynamics of the system is represented by the following equation:

$$
\frac{d x}{d t}=u(t)
$$

The initial and final conditions of the single state variable are:

$$
\left\{\begin{array}{l}
x(0)=0 \\
x(1)=1
\end{array} .\right.
$$


Once again, we denote the global minimum of the cost function by $J^{*}$. Meanwhile, let $u^{*}(t)$ and $x^{*}(t)$ denote the optimal control function and the optimal state variable, respectively. From analytical calculations, we have:

$$
\left\{\begin{array}{l}
u^{*}=t+\frac{1}{2} \\
J^{*}=\frac{23}{24}
\end{array} .\right.
$$

First of all, assume that the formation of the optimal control function is already known. Then we parameterize the optimal control function as a function of time using the following polynomial:

$$
u(t)=p_{2} t+p_{1},
$$

where both $p_{1}$ and $p_{2}$ vary in the same design space $[-2,2]$. According to the dynamics of the problem, the optimal state variable is also a polynomial with the form of:

$$
x(t)=\frac{1}{2} p_{2} t^{2}+p_{1} t+C .
$$

Based on the initial condition of the state variable, the constant $C$ can be determined as 0 . Meantime, based on the final condition of the state variable, an equation can be obtained as follows:

$$
\frac{1}{2} p_{2}+p_{1}=1
$$

which is considered as an equality constraint over the two parameters. Substituting the optimal control function and state variable into the original cost function generates a new function with the form of:

$$
J\left(p_{1}, p_{2}\right)=\frac{p_{1}\left(p_{1}+p_{2}+1\right)}{2}+\frac{p_{2}\left(p_{2}+1\right)}{6} .
$$

Secondly, the newly formed static optimization problem is solved using interval-related algorithms. In this example, the optimization process terminates when the widths of the intervals for the parameters and the parameterized cost function are all smaller than $1.00 \times 10^{-3}$. As can be seen in Table 2, the maximum widths of the intervals for the two parameters is about $0.52 \times 10^{-3}$ and the width of the interval for the cost function is around $1.00 \times 10^{-8}$.

Table 2. Interior and final results of Example 2.

\begin{tabular}{cccc} 
Iteration & Interval for $p_{1}$ & Interval for $p_{2}$ & Interal for $J$ \\
\hline 1 & {$[0.00000000,2.00000000]$} & {$[-2.00000000,2.00000000]$} & {$[0.33333333,0.96875001]$} \\
2 & {$[0.00000000,1.62500001]$} & {$[-1.25000001,2.00000000]$} & {$[0.77864583,0.95898438]$} \\
3 & {$[0.00000000,1.03125001]$} & {$[-0.06250001,2.00000000]$} & {$[0.91194661,0.95837403]$} \\
4 & {$[0.23437499,0.75781251]$} & {$[0.48437499,1.53125001]$} & {$[0.94664510,0.95833588]$} \\
5 & {$[0.36718749,0.63085938]$} & {$[0.73828124,1.26562501]$} & {$[0.95540555,0.95833493]$} \\
6 & {$[0.43359374,0.56591797]$} & {$[0.86816406,1.13281251]$} & {$[0.95760103,0.95833335]$} \\
$\vdots$ & $\vdots$ & $\vdots$ & $\vdots$ \\
15 & {$[0.49987052,0.50012948]$} & {$[0.99974106,1.00025896]$} & {$[0.95833333,0.95833334]$}
\end{tabular}

Finally, the interval polynomials obtained from interval-related algorithms are plotted in Fig. 8. On the top of Fig. 8, the optimal control function can be seen on the left and a small part is scaled up on the right. It is important to note that the optimum is not a single curve but an area enclosed by two curves and that the analytical optimum locates within the red area. On the bottom of Fig. 8, the optimal state variable is shown in the same way. Once again, the optimum given by analytical calculation is enclosed by the red area. 

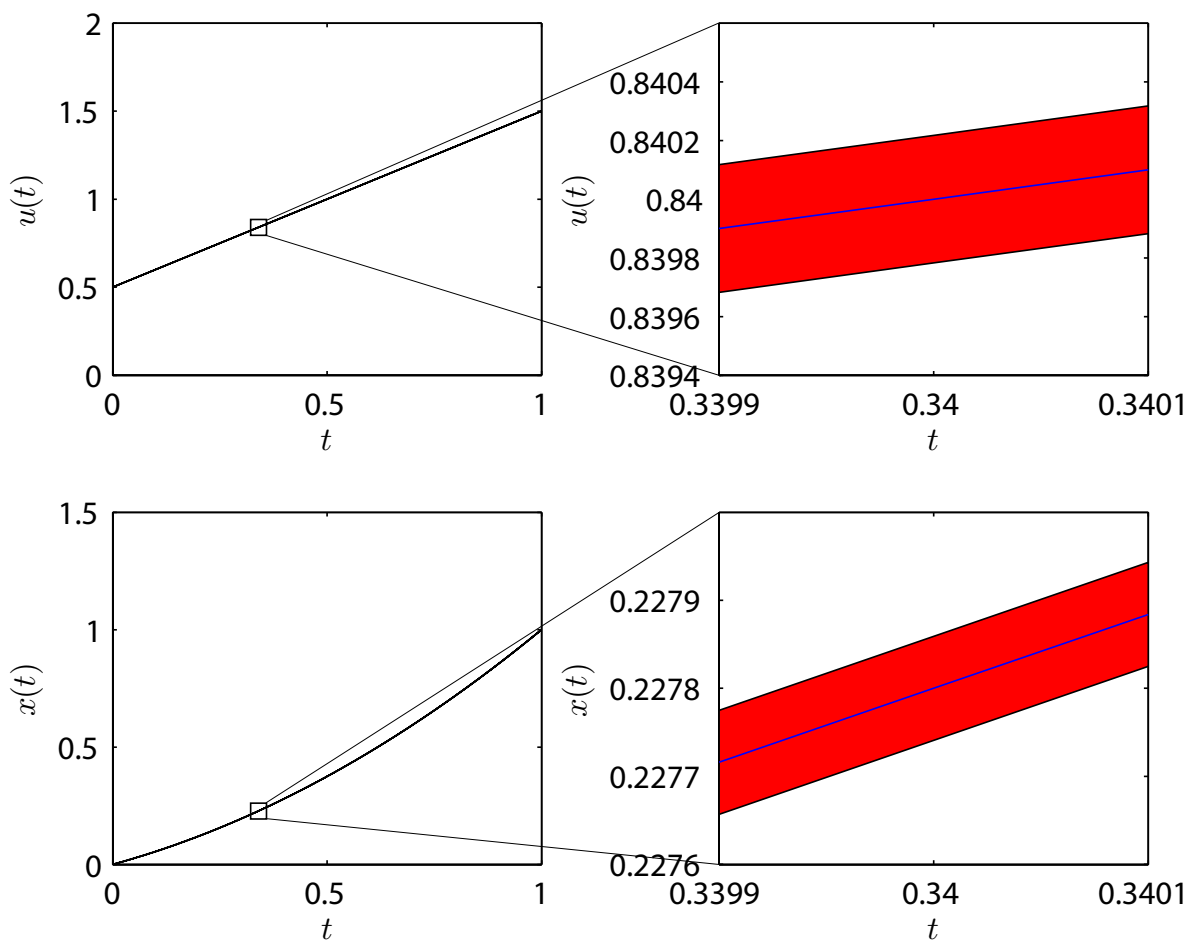

Figure 8. Example 2: the optimal control function and state variable.

\section{Example 3}

In the end, we build an optimization problem with the models given in Section II and solve it with the proposed interval-related algorithms. As we all know, arrival trajectory optimization for passenger aircraft is a dynamic optimization problem, so parameterization is again contained as a necessary method to finish the conversion into a static optimization problem. Differently, some of the state variables rather than the control functions are parameterized during the conversion. Details of the parameterization will be described in following papers.

\section{Simplified Point-Mass Equations of Motion}

As for a passenger aircraft, the movements of its center of gravity are governed by the point-mass equations of motion. Since the complexity of the equations affects the optimization and results significantly, a process of simplification should be carried out before proceeding further. Because the angle of attack $\alpha$ is always small, the trigonometric functions of this angle can be written as:

$$
\left\{\begin{array}{l}
\cos \alpha=1 \\
\sin \alpha=0
\end{array} .\right.
$$

After applying such assumptions to Eq.1, the simplified point-mass equations of motion has the form of:

$$
\left\{\begin{array}{rl}
\frac{d x}{d t} & =V_{T} \cos \gamma \\
\frac{d h}{d t} & =V_{T} \sin \gamma \\
\frac{d V_{T}}{d t} & =\frac{T\left(V_{T}, h\right)-D\left(V_{T}, h, \alpha\right)-m g \sin \gamma}{m} \\
\frac{d \gamma}{d t} & =\frac{L\left(V_{T}, h, \alpha\right)-m g \cos \gamma}{m V_{T}}
\end{array} .\right.
$$




\section{Reference Trajectory}

As mentioned in Section I, TDDA trajectories are designed to avoid the horizontal flight segments during arrivals and thus they already have improved performance compared with the traditional step-down approach trajectories. Therefore, a TDDA trajectory is selected as a reference trajectory in order to evaluate the results provided by the proposed optimization tool. Seen in Fig. 9, it is assumed that the aircraft navigates along a straight line from P0 to P4, during which the true airspeed decreases linearly and the engine throttles are all set at flight-idle or nearly flight-idle. The aircraft is operating with FLAPS- 5 and retracted landing-gears. Three interior operating points (P1, P2 and P3) are selected and thus the entire trajectory is divided into four segments. The distance to the runway threshold, the flight altitude, the true airspeed and the engine power at each of the five points are given in Table 3. With a population distribution model built by the authors, the number of awakenings caused by the TDDA trajectory is 3170 .

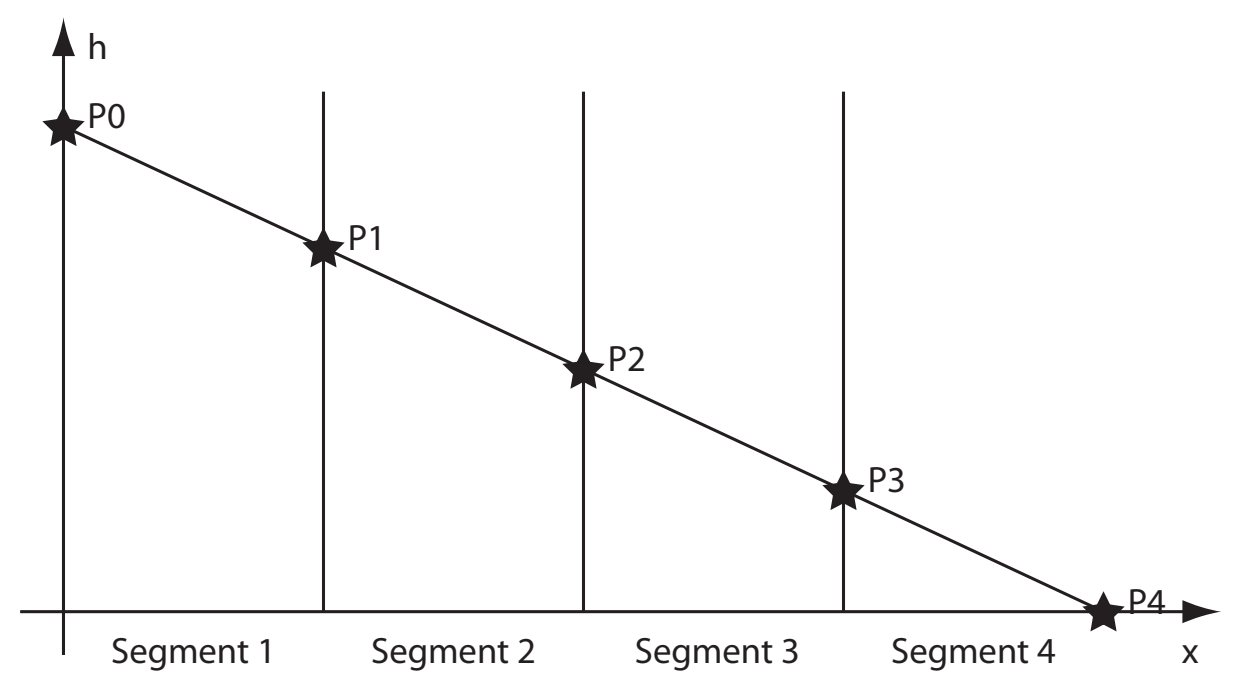

Figure 9. Example 3: part of a Three Degree Decelerating Approach (TDDA) trajectory.

Table 3. Example 3: flight data at all operating points of the TDDA trajectory.

\begin{tabular}{cccccc}
$\begin{array}{c}\text { Point } \\
(-)\end{array}$ & $\begin{array}{c}\text { Time } \\
(\mathrm{s})\end{array}$ & $\begin{array}{c}\text { Horizontal Distance to } \\
\text { Runway Threshold }(\mathrm{m})\end{array}$ & $\begin{array}{c}\text { Altitude } \\
(\mathrm{m})\end{array}$ & $\begin{array}{c}\text { True Airspeed } \\
(\mathrm{m} / \mathrm{s})\end{array}$ & $\begin{array}{c}\text { Engine Power } \\
(\mathrm{KN})\end{array}$ \\
\hline P0 & 0 & -29079.6523 & 1524.0000 & 100.6773 & 8.1534 \\
P1 & 30 & -26112.8379 & 1368.5158 & 97.3818 & 8.5896 \\
P2 & 60 & -23244.7529 & 1218.2059 & 94.0863 & 9.2128 \\
P3 & 90 & -20475.3973 & 1073.0701 & 90.7908 & 10.0542 \\
P4 & 124 & -17456.1471 & 914.8379 & 87.0559 & 11.3177
\end{tabular}

\section{Design Space and Parameters}

Design space is an important factor in the optimization problem. As shown in Fig. 10, the flight-path angle does not remain at three degrees any more due to the fact that the aircraft is allowed to descend in different manners. However, it is important to note that the aircraft could not descend too fast due to the requirements from aircraft performance, passenger comfort, and aviation regulations. These limits appear in the optimization process as constraints. As listed in Table 4, there are totally six parameters: (1) $p_{11}, p_{21}$ and $p_{31}$ with the unit of degrees represent the flight-path angles of the first three flight segments; (2) $p_{12}$, $p_{22}$ and $p_{32}$ with the unit of seconds represent the flight times required by the first three flight segments. 
The flight-path angle and flight time of the last fight segment are determined by the final conditions of the aircraft at $\mathrm{P} 4$.

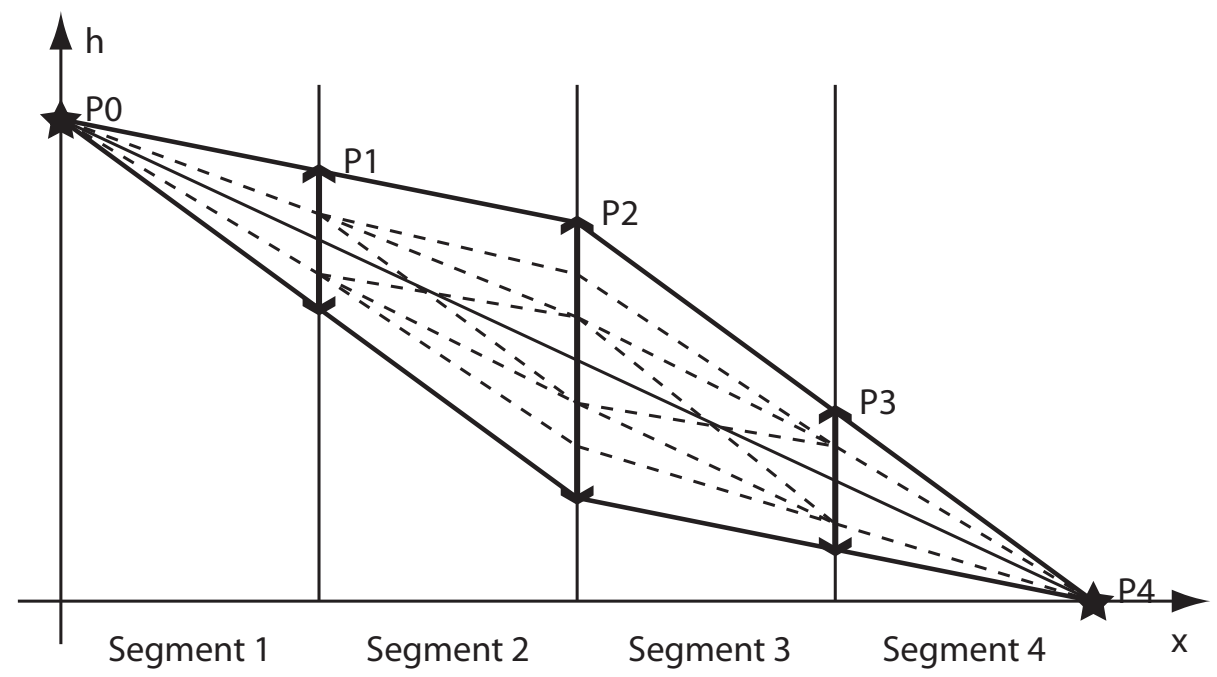

Figure 10. Example 3: design space for the defined optimization problem.

Table 4. Example 3: parameters in the optimization and their initial intervals.

\begin{tabular}{ccc} 
Parameters & Initial Interval & Width \\
\hline$p_{11}$ & {$[-5,-1]$} & 4 \\
$p_{21}$ & {$[-5,-1]$} & 4 \\
$p_{31}$ & {$[-5,-1]$} & 4 \\
$p_{12}$ & {$[-25,-35]$} & 10 \\
$p_{22}$ & {$[-25,-35]$} & 10 \\
$p_{32}$ & {$[-25,-35]$} & 10
\end{tabular}

\section{Optimization Results}

As can be seen in Table 5, the widths of the intervals for the parameters obtained from INTLAB are all extremely small. As a result, we can consider those intervals as real numbers and the error resulted from this approximation is ignorable in the practical problem. The distance to the runway threshold, the flight altitude, the true airspeed and the engine power at each of the five points are given in Table 6 . The flight data at the initial and final points are identical with those from the TDDA trajectory since we intended to evaluate the improvement provided by the optimization tool by comparing two trajectories. With the same population distribution model, the awakenings generated by the optimal trajectory from the optimization tool using interval analysis is 3166 which is slightly smaller than the value from the referenced TDDA trajectory.

\section{Conclusions}

This paper presents the arrival trajectory optimization problem for passenger aircraft, which is contained in the CleanEra project. Interval analysis is introduced as a preparation for the development of intervalrelated algorithms that have the capability of solving optimization problem globally. Two numerical examples from previous publications are given to demonstrate the applicability of the developed algorithms when they 
Table 5. Example 3: parameters in the optimization and the final intervals.

\begin{tabular}{ccc} 
Parameters & Initial Interval & Width \\
\hline$p_{11}$ & {$[-3.213400589343780,-3.213400589343778]$} & $2 \times 10^{-15}$ \\
$p_{21}$ & {$[-1.750000000000004,-1.750000000000002]$} & $2 \times 10^{-15}$ \\
$p_{31}$ & {$[-2.750000000000008,-2.750000000000007]$} & $1 \times 10^{-15}$ \\
$p_{12}$ & {$[30.978083061518070,30.978083061518070]$} & 0 \\
$p_{22}$ & {$[30.548171850145170,30.548171850145170]$} & 0 \\
$p_{32}$ & {$[30.351453266372928,30.351453266372928]$} & 0
\end{tabular}

Table 6. Example 3: flight data at all operating points of the optimal trajectory.

\begin{tabular}{ccccc}
$\begin{array}{c}\text { Point } \\
(-)\end{array}$ & $\begin{array}{c}\text { Horizontal Distance to } \\
\text { Runway Threshold }(\mathrm{m})\end{array}$ & $\begin{array}{c}\text { Altitude } \\
(\mathrm{m})\end{array}$ & $\begin{array}{c}\text { True Airspeed } \\
(\mathrm{m} / \mathrm{s})\end{array}$ & $\begin{array}{c}\text { Engine Power } \\
(\mathrm{KN})\end{array}$ \\
\hline P0 & {$[-29079.6523,-29079.6523]$} & {$[1524.0000,1524.0000]$} & {$[100.6773,100.6773]$} & {$[-7.0430,-7.0429]$} \\
P1 & {$[-26112.8379,-26112.8379]$} & {$[1357.4332,1357.4333]$} & {$[96.1671,96.1672]$} & {$[-4.8081,-4.8080]$} \\
P2 & {$[-23244.7529,-23244.7529]$} & {$[1280.8879,1280.8880]$} & {$[90.4083,90.4084]$} & {$[16.9377,16.9378]$} \\
P3 & {$[-20475.3973,-20475.3973]$} & {$[1085.1842,1085.1843]$} & {$[88.6099,88.6100]$} & {$[9.1514,9.1515]$} \\
P4 & {$[-17447.7914,-17447.7914]$} & {$[914.8379,914.8379]$} & {$[87.0559,87.0559]$} & {$[12.9620,12.9621]$}
\end{tabular}

are used to solve both static and dynamic optimization problems. As for the trajectory optimization problem, we can draw a conclusion that the proposed optimization tool is able to improve the performance of arrival trajectories of passenger aircraft in terms of noise nuisance.

There are a number of reasons that are able to explain why the improvement is not that significant. First of all, the TDDA trajectories do not include horizontal flight segments already and thus they are considered as optimal trajectories compared with the conventional step-down appraoch trajectories. Secondly, the trajectory appearing in the optimization process is just a small part of the entire arrival. In practice, the aircraft starts from a clean configuration and extends its flaps and landing-gears gradually. If the entire arrival is taken into account, larger improvement is expected from the optimization tool. Last but not least, the trajectory is a two-dimensional case which is rather simpler than the actual situation. If the trajectory is extended to a three-dimensional space, more parameters and a larger design space will yield more encouraging results.

\section{References}

${ }^{1} \mathrm{ICAO}$, Annex 16 -Environmental Protection, Volume 1, Aircraft Noise - to the Convention on International Civil Aviation, Fifth Edition, Jul. 2008.

${ }^{2}<$ http://www.cleanera.tudelft.nl/>

${ }^{3}$ Koeslag, M. F., Advanced Continuous Descent Approaches - An Algorithm Design for the Flight Management System, National Aerospace Laboratory, NLR-TR-200103142, Aug. 2001.

${ }^{4}$ Clark, J-P. B., System Analysis of Noise Abatement Procedures Enabled by Advanced Flight Guidance Technology, Journal of Aircraft, Vol. 37, No. 2, pp. 266-273, 2000.

${ }^{5}$ Visser, H. G., and Wijnen, R. A. A., Optimization of Noise Abatement Arrival Trajectories, AIAA Guidance, Navigation and Control Conference and Exhibit, Montreal, Canada, 6-9 Aug. 2001.

${ }^{6}$ Moore, R. E., Interval Analysis, Prientice Hall, Englewood Cliffs, N.J., 1966.

${ }^{7}$ Hansen, E. R., Global Optimization using Interval Analysis - the one dimensional case, Journal of Optimization Theory and Applications, Vol. 29, No. 3, pp. 331-344, 1979.

${ }^{8}$ Hansen, E. R., Global Optimization using Interval Analysis - the multiple-dimensional case, Numerical Mathematik, Vol. 34, No. 3, pp. 247-270, 1980.

${ }^{9}$ van Kampen, E., Chu, Q. P., and Mulder, J. A., Nonlinear Aircraft Trim using Interval Analysis, AIAA Guidance, Navigation and Control Conference and Exhibit, Hilton Head, South Carolina, 20-23 Aug. 2007.

${ }^{10}$ van Kampen, E., Zaal, P. M. T., de Weerdt, E., Chu, Q. P., and Mulder, J. A., Optimization of Human Perception 
Modeling using Interval Analysis, AIAA Guidance, Navigation and Control Conference and Exhibit, Honolulu Hawaii, 18-21 Aug. 2008.

${ }^{11}$ Filipe, N. P. S., de Weert, E., van Kampen, E., Chu, Q. P., and Mulder, J. A., Terminal Area Energy Management Trajectory Optimization using Interval Analysis, AIAA Guidance, Navigation and Control Conference and Exhibit, Chicago, Illinois, 20-23 Aug. 2007.

12 de Weert, E., Chu, Q. P., and Mulder, J. A., Neural Network Output Optimization using Interval Analysis, IEEE Transactions on Neural Networks, Vol. 20, No. 4, pp.638-653, 2009.

${ }^{13}$ Teengs, M., Model of the Boeing 747-400 with CF6-80C2B1F Engines, Exercise Report, Faculty of Aerospace Engineering, Delft University of Technology, Delft, 2003.

${ }^{14}$ Boeker, E. R., Dinges, E., He, B., Roof, C. J., Gerbi, P. J., Rapoza, A. S. and Hemann R. J., Integrated Noise Model (INM) Version 7.0 Technical Manual, jan. 2008

${ }^{15}$ FICAN, Effects of Aviation Noise on Awakenings from Sleep, Jun, 1997.

${ }^{16}$ Hansen E. R., Global Optimization using Interval Analysis, Marcel Dekker, Inc., New York, 1992.

${ }^{17}$ Hansen, E. R., and Walster, G. W., Global Optimization using Interval Analysis, 2nd Edition, Marcel Dekker, Inc., New York, 2004.

${ }^{18}$ Rump, S. M., Interval Computations with INTLAB, Brazilian Electronic Journal on Mathematics of Computation, Jan. 1999.

${ }^{19}$ Rump, S. M., INTerval LABoratory, Developments in Reliable Computing, Kluwer Academic Publishers, pp. 77-104, 1999.

${ }^{20}<$ http://www.ti3.tu-hamburg.de/rump/intlab/> . 